\title{
Abdominal pain and jaundice: Beware of the perforated appendix
}

\author{
Abeysekera WYM, De Silva WDD, Chandrapalan S, Pragatheswaran P, Banagala ASK, \\ Colombo South Teaching Hospital, Kalubowila, Sri Lanka
}

Correspondence: Dr.W.Y.M. Abeysekera; (yohan.abeysekera@yahoo.com)

\section{Introduction}

Acute Appendicitis is a common surgical condition with myriad clinical presentations which often present a diagnostic challenge to the clinician. This report discusses an unusual presentation of appendicitis and its causation.

\section{Case Report}

A 29-year-old male presented with gradually increasing lower abdominal pain for 3 days duration. He had not opened bowel for 2 days and had one episode of non-bilious vomiting. He had a history of bathing in a natural water tank in an area endemic for leptospirosis 2 weeks previously. He had no history of liver disease, haemolytic disease or risk factors for hepatitis B and C.

On examination he was afebrile but clearly icteric. Bilateral conjunctival injections were noted (which the patient claimed was due to an allergic condition present prior to the current illness). Pulse rate of $100 / \mathrm{min}$ and blood pressure of $100 / 60 \mathrm{mmHg}$ were recorded. Abdomen was mildly distended with deep lower abdominal tenderness which was marked over the left iliac fossa. Faeces were present on rectal examination. Urine was dark yellow in colour and passed adequately. There was no significant myalgia.

Six hours after admission the patient developed a high fever spike with chills and rigors. His WBC count was $14.2 \times 10^{9} / \mathrm{L}$ with an $87 \%$ neutrophil count. The platelet count was well over $200 \times 10^{9} / \mathrm{L}$. Total bilirubin was elevated $(189 \mu \mathrm{mol} / \mathrm{L})$ with marginally high liver enzyme values (AST $105 \mathrm{U} / \mathrm{L}$ and ALT $80 \mathrm{U} / \mathrm{L}$ ). Abdominal radiograph revealed distended small bowel loops with multiple air-fluid levels and USS abdomen demonstrated mild hepatomegaly with no free fluid with a suggestion of possible sub acute intestinal obstruction. Given the equivocal clinical findings, a medical opinion was sought regarding the possibility of leptospirosis and the patient was commenced on management for leptospirosis.

Over the next 24 hours there was deterioration of the clinical parameters of the patient with increasing tachycardia, tachypnoea and abdominal distension. Abdominal tenderness persisted over the left lilac fossa region. The patient underwent exploratory laparotomy at which a perforated appendix with para appendicular and retro-cecal abscesses were found. Appendicectomy was performed and the abscess drained. The cultures from the peritoneal fluid were positive for E-coli. The patient recovered well and serum bilirubin level returned to normal 14 days after the surgery.

\section{Discussion}

Acute appendicitis is the commonest abdominal pathology leading to emergency surgery both in paediatric and adult age groups. The overall time risk of developing appendicitis is approximately $7-12 \%$ (1). The morbidity and mortality associated with appendicitis increase significantly with perforation.

Overt clinical jaundice is not a finding usually associated with acute appendicitis and prompted the initial misdiagnosis in this patient. The association between appendicitis and jaundice or hyperbilirubinaemia however, has been noted for over 50 years, essentially in patients with appendiceal perforation (2).

Despite the availability of modern diagnostic tools delays in diagnosing or misdiagnosis of perforated appendix is not uncommon. Some recent studies showed the strong possibility of hyperbilirubinaemia in perforated or necrotizing appendicitis (3) in which they have suggested it as a predictor of perforation. 
In a retrospective study, a higher specificity was demonstrated for hyperbilirubinemia in perforated appendicitis than WBC counts and CRP levels (4). Other authors have suggested that high bilirubin level maybe a better discriminator between acute suppurative appendicitis and perforated appendicitis than WBC count (5) although CRP appears superior to both (6).

The cause of hyperbilirubinemia in acute appendicitis is the subject of some conjecture. It is well known that hyperbilirubinaemia can occur in non-hepatic bacterial infections especially in intraabdominal sepsis. Inflammatory cytokines induced by infection can be potent mediators of intra-hepatic cholestasis (7). However sepsis alone may not be the only explanation for jaundice in appendicitis given its early appearance in the course of pathogenesis. Therefore could it be due to the organism involved in the appendicitis?

Miller and Irvine have shown a correlation with E-coli positive cultures in patients with acute appendicitis and jaundice (2).

E-coli being the commonest pathogen related to appendicitis, several recent studies in rat liver models showed its effect on hepatic microcirculation and sinusoidal damage. The E-coli endotoxin appeared to impair hepatocyte uptake and secretion of bile acids as well as impact on normal biliary flow (4-5). E-coli induced haemolysis increasing bilirubin load is also an additional factor which may aggravate the condition (4-5).

\section{Key Learning Points}

- Jaundice in acute appendicitis is an uncommon finding which can lead to a misdiagnosis.

- If jaundice is present in a patient with acute appendicitis, it is most likely to be a perforated appendix.

- Role of E-Coli in development of jaundice.

\section{References}

1. Chen LE, Buchman TG. Acute abdominal pain and appendicitis. In: Klingensmith ME, Chen LE, Glasgow SC, Goers TA, Melby S, eds. The Washington Manual of Surgery. $5^{\text {th }}$ Edition. Lippincott, Williams \& Wilkins, 2008: 200-7.

2. Miller DF, Irvine RW. Jaundice in acute appendicitis. Lancet 1969; 1:321-3.

3. Estrada JJ, Petrosyan M, Barnhart J, et al. Hyperbilirubinemia in appendicitis: a new predictor of perforation. Journal of Gastrointestinal Surgery 2007; 11: 714-8.

4. Sand M, Bechara FG, Holland-Letz T, et al. Diagnostic value of hyperbilirubinemia as a predictive factor for appendiceal perforation in acute appendicitis. American Journal of Surgery 2009; 198: 193-8.

5. Atahan K, Üreyen O, Aslan E, et al: Preoperative Diagnostic Role of Hyperbilirubinaemia as a Marker of Appendix Perforation. The Journal of International Medical Research 2011;39: 609-18.

6. Beltran MA, Mendez PE, Barrera RE et al. Is hyperbilirubinaemia in appendicitis a better predictor of perforation than C-reactive protein? - a prospective study. Indian Journal of Surgery 2009; 71:265-72.

7. Trauner M, Fickert P, Stauber RE. Inflammation-induced cholestasis. Journal of Gastroenterology and Hepatology 1999; 14: 946-59. 